\title{
Preventing surgical site infection after Lichtenstein inguinal hernioplasty: Preliminary results about local versus IV antibiotics prophylaxis from a low- income country university hospital.
}

\section{Patrick Charlorin ( $\nabla$ charlorin3816@gmail.com )}

General surgery, Saint Damien Pediatric Hospital of Haiti https://orcid.org/0000-0002-2915-5562

\section{Paul Reginald CADET}

General Surgery, Hospital of the State University of Haiti

\section{Research Article}

Keywords: Inguinal hernia, Surgical site infection, Antibiotics prophylaxis

Posted Date: August 26th, 2021

DOl: https://doi.org/10.21203/rs.3.rs-841767/v1

License: (c) (1) This work is licensed under a Creative Commons Attribution 4.0 International License. Read Full License 


\section{Abstract}

BACKGROUND: Preventing Surgical site infection (SSI) after Liechtenstein hernioplasty is an important step in the management of inguinal hernia. In this study, we present the preliminary result about the use of IV ceftriaxone prophylaxis vs the local mesh-soaked gentamicin prophylaxis for the prevention of SSI after Liechtenstein hernioplasty.

METHOD: We conducted a randomized controlled study in 2014, at the state university hospital. We evaluated the occurrence of SSI on 44 patients with an inguinal hernia who underwent an elective Liechtenstein repair using a polypropylene mesh. 13 patients received IV ceftriaxone prophylaxis. The remaining 31 received local mesh-soaked gentamicin prophylaxis. The patients were followed for infection and other complications by an independent surgeon on days 3,10 , and 30 .

RESULTS: The mean age of our patients was $48 \mathrm{y}$ for the IV prophylaxis group and $44 \mathrm{y}$ for the local prophylaxis group. 9 were located on the right side in the IV prophylaxis group while 32 were located on the right side for the local prophylaxis group. $30,6 \%$ of our patients had associated conditions in the IV prophylaxis group vs $13 \%$ in the local prophylaxis group. We found no SSI at the time of the study for either the IV or local prophylaxis group. One case of inguinal pain was observed in the local prophylaxis group.

CONCLUSION: This study indicates that local mesh-soaked gentamicin prophylaxis is as effective as IV ceftriaxone prophylaxis in preventing surgical site infection after Liechtenstein hernioplasty. But further evidence needs to be established.

\section{Background}

The inguinal hernia has become one of the leading causes of disability and work loss[1]. More than one million inguinal hernia repairs are performed each year in the USA and Europe[2]. Lichtenstein tensionfree hernioplasty remains one of the most popular technics for the management of inguinal hernia in adults [1]. The use of antibiotic prophylaxis is a routine when it comes to practice surgery using prosthetics[2], for the occurrence of an infection can result in several issues including an increase in the length of hospitalization and cost, even the need to remove the prosthetic[3], [4]. A. Pardhan, S. Mazahir, A. R. Alvi, et al have found that the incidence of surgical site infection after hernia repair with mesh in a daycare setting at a tertiary care hospital of a low-income country was higher than internationally reported 1-4\% incidence[1], [2]. In the case of hernioplasty, some studies have been discussing whether it was better to use antibiotics prophylaxis intravenously or locally to prevent superficial and deep surgical sites infection after Lichtenstein hernioplasty[5]. Study shows that local antibiotics are equivalent to intravenous antibiotics in the prevention of surgical site infection in inguinal hernioplasty[5]. Here in Haiti in our knowledge, there is no data about SSI after hernioplasty or the use of antibiotics for the prevention of SSI in inguinal hernioplasty. In this study, we present our preliminary result about the use of intravenous or local antibiotics for the prevention of SSI after Lichtenstein hernioplasty. 


\section{Materials And Method}

We conducted a randomized controlled study in 2014, at the state university hospital. We evaluated the occurrence of SSI on 44 patients with an inguinal hernia who underwent an elective Liechtenstein repair using a polypropylene mesh. The inclusion criteria were age $>18$ and $<80$ years, unilateral inguinal or inguinoscrotal hernia with American Society of Anesthesiology Classification (ASA) I and II, and primary elective cases. The exclusion criteria were age $>80$ years, immunocompromising patients, recurrent hernia, bilateral hernia, strangulated or incarcerated hernia, femoral hernia. Inform consent was obtained from all the patients and they were randomized to receive either $1 \mathrm{~g}$ of intravenous ceftriaxone or $80 \mathrm{mg}$ gentamycin used as a local antibiotic. The mesh was soaked in $80 \mathrm{mg}$ of gentamycin diluted in $20 \mathrm{ml}$ of normal saline and the inguinal canal was irrigated with the gentamycin solution. The patients were followed for infection and other complications by an independent surgeon on days 3,10 , and 30 . Surgical Site infections were defined according to the Center for Disease Control. An excel data sheet was created to collect the patient's information. Data analyses were conducted using excel. This study was approved by the ethics committee of the hospital.

\section{Results}

During the period of March 2014 and July 2014, a total of 44 patients were enrolled in the study. After randomization 31 (70.4\%) patients were assigned to local mesh soak antibiotic prophylaxis, while the remaining 13 (29.6\%) patients were assigned to intravenous antibiotic prophylaxis.

For the local mesh soak antibiotic prophylaxis group $29(93,5 \%)$ were males, The mean age of 44 years old, with an age bracket of 22 to 64 years old. For the IV antibiotic prophylaxis group all the $13(100 \%)$ patients were males, with a mean age of $48 y e a r s$ old and an age bracket of 19 to 73 years old.

Table 1: Baseline and operative characteristics of the patients: 


\begin{tabular}{|c|c|c|}
\hline Characteristic & $\begin{array}{l}\text { IV antibiotic prophylaxis } \\
\text { group } \\
(\mathrm{n}=13)\end{array}$ & $\begin{array}{l}\text { Local mesh soak antibiotic } \\
\text { prophylaxis group } \\
(\mathrm{n}=31)\end{array}$ \\
\hline Age (y) & $48(19-73)$ & $44(22-64)$ \\
\hline \multicolumn{3}{|l|}{ Sex } \\
\hline Male & $13(100 \%)$ & $29(93,5 \%)$ \\
\hline Female & $0(0 \%)$ & $2(6,5 \%)$ \\
\hline \multicolumn{3}{|l|}{ Hernia characteristic } \\
\hline Right & $9(69,2 \%)$ & $32(73 \%)$ \\
\hline Left & $4(30,8 \%)$ & $12(27 \%$ \\
\hline \multicolumn{3}{|l|}{ Comorbidities (n, \%) } \\
\hline Diabetes & $1(7,6 \%)$ & $0(0 \%)$ \\
\hline Hypertension & $3(23 \%)$ & $4(13 \%)$ \\
\hline None & $9(69,4 \%)$ & $27(87 \%)$ \\
\hline \multicolumn{3}{|l|}{ Type of Incision (n, \%) } \\
\hline Oblique & $3(23 \%)$ & $2(6,4 \%)$ \\
\hline Horizontal & $10(77 \%)$ & $29(93,6 \%)$ \\
\hline $\begin{array}{l}\text { Operation duration (mean, } \\
\text { minutes) }\end{array}$ & $80(45-120)$ & $100(65-120)$ \\
\hline
\end{tabular}

Most of the patients of our study present with a right inguinal or inguinoscrotal hernia, $69.2 \%$ of the patients benefit from the IV antibiotic prophylaxis, and $73 \%$ of the patients benefit from the local mesh soak antibiotic prophylaxis. A horizontal inguinal incision was performed for most of the patients in our study. $77 \%$ for the IV antibiotic prophylaxis group and $93 \%$ for the local mesh soak antibiotic prophylaxis. Comorbidities identify for our patients were hypertension in 7 patients and diabetes in one patient. For the IV antibiotic prophylaxis, we had $7.6 \%$ of the patients being hypertensive and one patient was known to have type 2 diabetes. For the local mesh soak antibiotic prophylaxis, we found that $13 \%$ had comorbidities consisting of hypertension. The mean duration of surgery was $80 \mathrm{mns}$ for the patients who benefit from the IV antibiotic prophylaxis, and it was $100 \mathrm{mns}$ for the patients who benefit from the local mesh soak antibiotic prophylaxis.

After one month of follow-up, no surgical site infection was identified in either group of our study. Other complications like hematoma, seroma, or hernia recurrence had been investigated but we didn't find any of them. One patient of the local mesh soak antibiotic prophylaxis reported having pain at the site of surgery but we found no sign of infection.

Table 2: Incidence of postoperative complications after one month of follow up: 


\begin{tabular}{cll}
\hline \hline Complication & $\begin{array}{l}\text { IV } \\
\text { antibiotic } \\
\text { prophylaxi } \\
\text { s group } \\
\text { (n= 13) }\end{array}$ & $\begin{array}{l}\text { Local mesh soak antibiotic } \\
\text { prophylaxis group } \\
\text { (n= 31) }\end{array}$ \\
\hline \hline Surgical site infection \\
$\begin{array}{c}\text { Superficial } \\
\text { Deep }\end{array}$ & 0 & 0 \\
Other complication & 0 & 0 \\
Hematoma & 0 & 0 \\
Seroma & 0 & 0 \\
Pain & 0 & 1 \\
Recurrence & 0 & 0 \\
Mortality & 0 & 0 \\
\hline \hline
\end{tabular}

\section{Discussion}

Since Lichtenstein has popularized his technic for inguinal hernia repair in 1984, It quickly becomes 4 years later the gold standard to evaluate the other technical hernia repair[6][7][8] A large range of mesh have been created[9] One of the principal reason of this advancement is that studies show a lower rate of recurrence with mesh with no difference in adverse effect[8][10]. When compared to Shouldice technic, the best non mesh actual open technic, the recurrence rate is still lower for Lichtenstein hernioplasty, with a similar rate of pain occurrence[8].

Infection after hernioplasty is a rare complication, but when it occurs it can be very dangerous. The reported incidence of mesh-related infection following hernia repair varies from 1 to $8 \%$, and the need to remove the mesh for the management of mesh infection was reported to be $0,13 \%$. In a very large report conduct by $\mathrm{H}$. Johanet, $\mathrm{N}$. Contival about the management of mesh infection after inguinal hernia repair, a rate of infection of 0,07 was reported. They also report that for the 38 patients who were managed for mesh infection, 15 patients required primary removal of the mesh. The remaining 23 underwent conservative treatment. (18 patients) $78,2 \%$ of those failed to the conservative management and required the removal of the mesh[10].

While Intravenous antibiotics and mesh excision are the keystones in the management of mesh infections, there are some specificities according to the type of mesh that has been used. In case of infection with polyester or polypropylene, some studies recommend drainage and the use of antimicrobial agents. But if the infection involves an extended polytetrafluoroethylene mesh, the removal of the mesh could be the definitive treatment[10].

The use of antibiotics for the reduction of mesh infection after hernioplasty is very controversial[3]. E. Just et al. reported a $0,2 \%$ case of SSI for patients who receive antibiotic prophylaxis after hernioplasty, in comparison to $1,2 \%$ of cases of SSI for patients who did not receive any antibiotic prophylaxis.[8] More 
interestingly Yerdel et al showed in a similar study a tenfold difference of infection rate $(0,7 \%$ in antibiotic arm vs $9 \%$ in the placebo arm)[5]. According to Aufenacker et al, there was no difference between the antibiotic prophylaxis group or the placebo group of patients who benefit from primary inguinal hernioplasty. In their study, they conclude that in Lichtenstein inguinal hernia repair, antibiotics prophylaxis is not indicated in low-risk patients[6]. Mazaki et al in a meta-analysis suggest that prophylactic antibiotic is efficacious for the prevention of SSI after open mesh hernia repair[2].

The use of intravenous antibiotic prophylaxis can be risky for the patients, anaphylaxis, hypersensitivity, interstitial nephritis is part of those risk. This justifies the idea of looking for an alternate way to conduct antibiotic prophylaxis, as the local route used in our study.

As the result is very limited with his low power. We can continue the investigation to more patients to see if we can find more data's supporting the use of local mesh-soaked antibiotics for the prevention of infection after Lichtenstein hernioplasty.

\section{Conclusion}

This is one of the first studies attempting to assess the use of local mesh soak antibiotic prophylaxis and the IV antibiotic prophylaxis for the prevention of SSI after inguinal Lichtenstein hernioplasty in Haiti, For the period of this study we found no difference in the outcome of our patients regarding the occurrence of SSI. But further evidence needs to be established.

\section{Declarations}

Competing interests: The authors declare no competing interests.

\section{References}

1. A. Pardhan, S. Mazahir, A. R. Alvi, and G. Murtaza, "Surgical site infection following hernia repair in the day care setting of a developing country: A retrospective review," J. Pak. Med. Assoc., vol. 63, no. 6, pp. 760-762, 2013.

2. T. Mazaki, K. Mado, H. Masuda, and M. Shiono, "Antibiotic prophylaxis for the prevention of surgical site infection after tension-free hernia repair: A bayesian and frequentist meta-analysis," J. Am. Coll. Surg., vol. 217, no. 5, pp. 788-801.e4, 2013, doi: 10.1016/j.jamcollsurg.2013.07.386, http://dx.doi.org/10.1016/j.jamcollsurg.2013.07.386.

3. T. Mazaki, K. Mado, H. Masuda, M. Shiono, N. Tochikura, and M. Kaburagi, "A randomized trial of antibiotic prophylaxis for the prevention of surgical site infection after open mesh-plug hernia repair," Am. J. Surg., vol. 207, no. 4, pp. 476-484, 2014, doi: 10.1016/j.amjsurg.2013.01.047, http://dx.doi.org/10.1016/j.amjsurg.2013.01.047.

4. G. Salamone et al., "Deep SSI after mesh-mediated groin hernia repair: Management and outcome in an Emergency Surgery Department," G. di Chir., vol. 38, no. 1, pp. 41-45, 2017, doi: 
10.11138/gchir/2017.38.1.041.

5. S. Praveen and M. Rohaizak, "Local antibiotics are equivalent to intravenous antibiotics in the prevention of superficial wound infection in inguinal hernioplasty," Asian J. Surg., vol. 32, no. 1, pp. 59-63, 2009, doi: 10.1016/S1015-9584(09)60011-7, http://dx.doi.org/10.1016/S10159584(09)60011-7.

6. T. J. Aufenacker et al., "The role of antibiotic prophylaxis in prevention of wound infection after lichtenstein open mesh repair of primary inguinal hernia: A multicenter double-blind randomized controlled trial," Ann. Surg., vol. 240, no. 6, pp. 955-961, 2004, doi:

10.1097/01.sla.0000145926.74300.42.

7. H. Johanet, N. Contival, and C. Coelio, "Infection profonde et cure de hernie inguinale par prothèse," J. Chir. Viscerale, vol. 148, no. 5, pp. 443-446, 2011, doi: 10.1016/j.jviscsurg.2011.09.005.

8. E. Just, X. Botet, S. Martínez, D. Escolà, I. Moreno, and E. Duque, "Reduction of the complication rate in Liechtenstein hernia repair," Int. J. Surg., vol. 8, no. 6, pp. 462-465, 2010, doi: 10.1016/j.ijsu.2010.06.006, http://dx.doi.org/10.1016/j.ijsu.2010.06.006.

9. R. Narkhede, N. M. Shah, P. R. Dalal, C. Mangukia, and S. Dholaria, "Postoperative Mesh InfectionStill a Concern in Laparoscopic Era," Indian J. Surg., vol. 77, no. 4, pp. 322-326, 2015, doi: 10.1007/s12262-015-1304-x.

10. H. Johanet, N. Contival, and Coelio Club, "Mesh infection after inguinal hernia mesh repair.," J. Visc. Surg., vol. 148, no. 5, pp. 78-80, 2011, doi: 10.1016/j.jviscsurg.2011.09.005. 